\title{
The Effects of Moxifloxacin on QTe Interval in Healthy Korean Male Subjects
}

\author{
Seol Ju Moon · Jongtae Lee $\cdot$ Hyungmi An $\cdot$ \\ Dong-Seok Yim $\cdot$ Jae-Yong Chung . \\ Kyung-Sang Yu $\cdot$ Joo-Youn Cho $\cdot$ Kyoung Soo Lim
}

Published online: 2 April 2014

(C) The Author(s) 2014. This article is published with open access at Springerlink.com

\begin{abstract}
Background and objective Moxifloxacin $400 \mathrm{mg}$ is a widely used positive control in thorough QT (TQT) studies, but its QT-prolonging effects in Korean subjects have not been studied. The present study was conducted to collect pilot data in Korean subjects after moxifloxacin administration to evaluate the adequacy of moxifloxacin as a positive control.

Methods Thirty-eight, healthy, Korean, male subjects were recruited for pharmacokinetic (PK) blood sampling and electrocardiography (ECG) recordings at three different study sites. On day 1, a baseline 12-lead ECG was recorded, and on day 2, ECG recordings were conducted after placebo, or moxifloxacin 400- or 800-mg administration. Baseline-corrected, placebo-adjusted, corrected QT $(\Delta \Delta \mathrm{QTc})$ values were calculated. Blood samples were
\end{abstract}

S. J. Moon - J. Lee $\cdot$ H. An · K.-S. Yu · J.-Y. Cho .

K. S. Lim $(\bowtie)$

Department of Clinical Pharmacology and Therapeutics, Seoul National University College of Medicine and Hospital,

101 Daehak-ro, Jongno-gu, Seoul, Korea

e-mail:kslim96@snu.ac.kr

H. An

Department of Statistics, Seoul National University, Seoul,

Korea

D.-S. Yim

Department of Clinical Pharmacology and Therapeutics, Seoul St. Mary's Hospital, The Catholic University of Korea, Seoul, Korea

J.-Y. Chung

Department of Clinical Pharmacology and Therapeutics, Seoul National University College of Medicine and Bundang Hospital, 300 Gumi-dong, Bundang-gu, Songnam, Gyeonggi-do, Korea collected after moxifloxacin administration and PK parameters were assessed.

Results A total of 33 subjects completed the study. The largest time-matched $\Delta \Delta \mathrm{QTc}$ occurred approximately $4 \mathrm{~h}$ after dosing, with $\Delta \Delta \mathrm{QTcI}$ (QT interval corrected by individual QT-RR regression model) values of $11.66 \mathrm{~ms}$ (moxifloxacin $400 \mathrm{mg}$ ) and $20.96 \mathrm{~ms}(800 \mathrm{mg}$ ). The mean and $90 \%$ confidence intervals of $\Delta \Delta \mathrm{QTCI}$ did not include zero at any of the measurement time points. There was a positive correlation between plasma moxifloxacin concentration and $\Delta \Delta \mathrm{QTCI}(r=0.422)$. Dose-proportional PK profiles were observed.

Conclusion Moxifloxacin $400 \mathrm{mg}$ is an adequate positive control in Korean TQT studies. Our results indicate that moxifloxacin $400 \mathrm{mg}$ can be used to evaluate the cardiac safety of a drug in Korean subjects.

\section{Introduction}

Currently, the International Conference on Harmonization of Technical Requirements for Registration of Pharmaceuticals for Human Use (ICH) recommends sponsors submitting new drug applications to evaluate the drug's effects on cardiac repolarization by conducting a clinical thorough QT (TQT) study [1]. This recommendation is set to investigate possible drug-induced prolongation of the QT interval and to prevent associated potentially fatal pro-arrhythmias, such as torsades de pointes. This growing concern for cardiac safety is because some drugs, which were not originally developed to treat cardiovascular diseases, were found to cause arrhythmias and were withdrawn from the market [2]. Since its publication in 2005, ICH guideline E14 has gained a substantial amount of interest, and the guideline's 
proposal to examine TQT is currently followed worldwide [3].

Although ICH guideline E14 does not specify the use of moxifloxacin as a positive control, it has been the most widely and most commonly used positive control in TQT studies [3]. The effects of moxifloxacin on QT interval have been well documented [4] and compared with ibutilide, an intravenous formulation that is the only other positive control that has been used in published TQT studies, moxifloxacin is orally administered and is therefore a better choice for use in blinded studies. When ICH guideline E14 was introduced in 2005, the initial assumption was that ethnicity would not significantly affect the results of TQT studies [5]. Since then, clinical data challenging this assumption have been accumulating. Unfortunately, two limitations have arisen to date: limited data evaluating inter-ethnic differences in baseline, drug-free QT intervals exist and evidence from TQT studies has been collected mostly from Caucasian subjects or subjects that do not adequately represent ethnic differences [5].

A known debate concerning which QT interval correction method should be used in TQT studies also exists [6]. QT intervals are influenced by the individual's heart rate and should be corrected (heart rate-corrected QT; QTc) for investigational purposes. Formulae that reflect individual heart rate include Bazett's formula, Fridericia's formula, and a correction using the individual QT/RR regression model. There was previously no consensus regarding which method to use in TQT studies [6], but as the data accumulated, it is now encouraged that newer correction formulae such as individual correction should be used [1]. In addition, TQT studies may use either the time-matched baseline method or the pre-dose baseline method. ICH guideline E14 recommends the use of the time-matched method for parallel studies and the use of the pre-dose method for crossover studies [1]; however, few studies have addressed the differences between the two baseline measurement methods. Comparing the two methods may provide some insight into whether using different baseline measurement methods significantly affects the results of TQT studies.

At present, no comparable published data collected from Korean subjects exist that can be used to evaluate an investigational product's effects on QT interval during the drug development phase. Furthermore, the effects of moxifloxacin 400 or $800 \mathrm{mg}$ (supratherapeutic dose) on QT prolongation have not been fully assessed in healthy Korean subjects, nor has the known diurnal variation been evaluated in this population [4]. Hence, an investigation is required to evaluate whether the usual positive control dose for TQT studies, moxifloxacin $400 \mathrm{mg}$, is adequate for Korean subjects and to determine whether moxifloxacin can be used as a positive control in Koreans, as outlined by
ICH guideline E14. Therefore, the aims of the present study were to evaluate QTc prolongation in healthy Korean male subjects (both at therapeutic and supratherapeutic doses of moxifloxacin), to assess the use of moxifloxacin as an adequate positive control, to compare QT interval correction methods, and to compare baseline measurement methods in Korean subjects.

\section{Methods}

\subsection{Subjects}

Healthy Korean male subjects, aged 20-40 years with body weight over $50 \mathrm{~kg}$ and within $\pm 20 \%$ of ideal body weight (calculated as: (height in $\mathrm{cm}-100) \times 0.9$ ), were recruited to participate in this study and written informed consent was obtained prior to participation. Eligibility was assessed based on medical history, physical examination, and laboratory test results (hematology, routine clinical chemistry, urinalysis, and serology, including hepatitis $\mathrm{B}$ and $\mathrm{C}$, and HIV) at screening. Twelve-lead electrocardiography (ECG) was taken at screening and subjects with ECG findings, such as QTc interval using Fridericia's formula (QTcF) over $450 \mathrm{~ms}$, PR interval above $200 \mathrm{~ms}$ or below $110 \mathrm{~ms}$, intraventricular conduction delay with QRS over $120 \mathrm{~ms}$, second- or third-degree atrioventricular block, pathologic Q waves (defined as Q-wave over $40 \mathrm{~ms}$ or depth over $0.5 \mathrm{mV}$ ), ventricular preexcitation, and left or right bundle branch block, were excluded from the study. Subjects with the following criteria were also excluded: family history of long QT syndrome, any torsades de pointes risk factors, such as sudden death, cardiac failure, hypokalemia, and arrhythmia, and history of hypersensitivity to drugs, including quinolone antibiotics. Enrolled subjects were asked not to drink alcohol or caffeinated beverages and not to smoke from $24 \mathrm{~h}$ prior to hospitalization until the end of the study.

\subsection{Study Design}

This study was designed as a multi-center, randomized, open-label, placebo-controlled, three-way crossover trial. Eligible subjects were randomized into six sequences (Fig. 1).

On day 1, baseline 12-lead ECGs were measured after $10 \mathrm{~min}$ of supine position using either MAC5000 or MAC5500 (GE Healthcare, Milwaukee, WI, USA; set at $25 \mathrm{~mm} / \mathrm{s}$ ) at the following time points: $0,1,2,3,4,6,8,12$, 16, and $24 \mathrm{~h}$. ECGs were recorded once for every time point. On day 2, subjects received one of the three treatments in each period according to their sequence group: placebo (water), moxifloxacin $400 \mathrm{mg}$ (Avelox Tablets, 


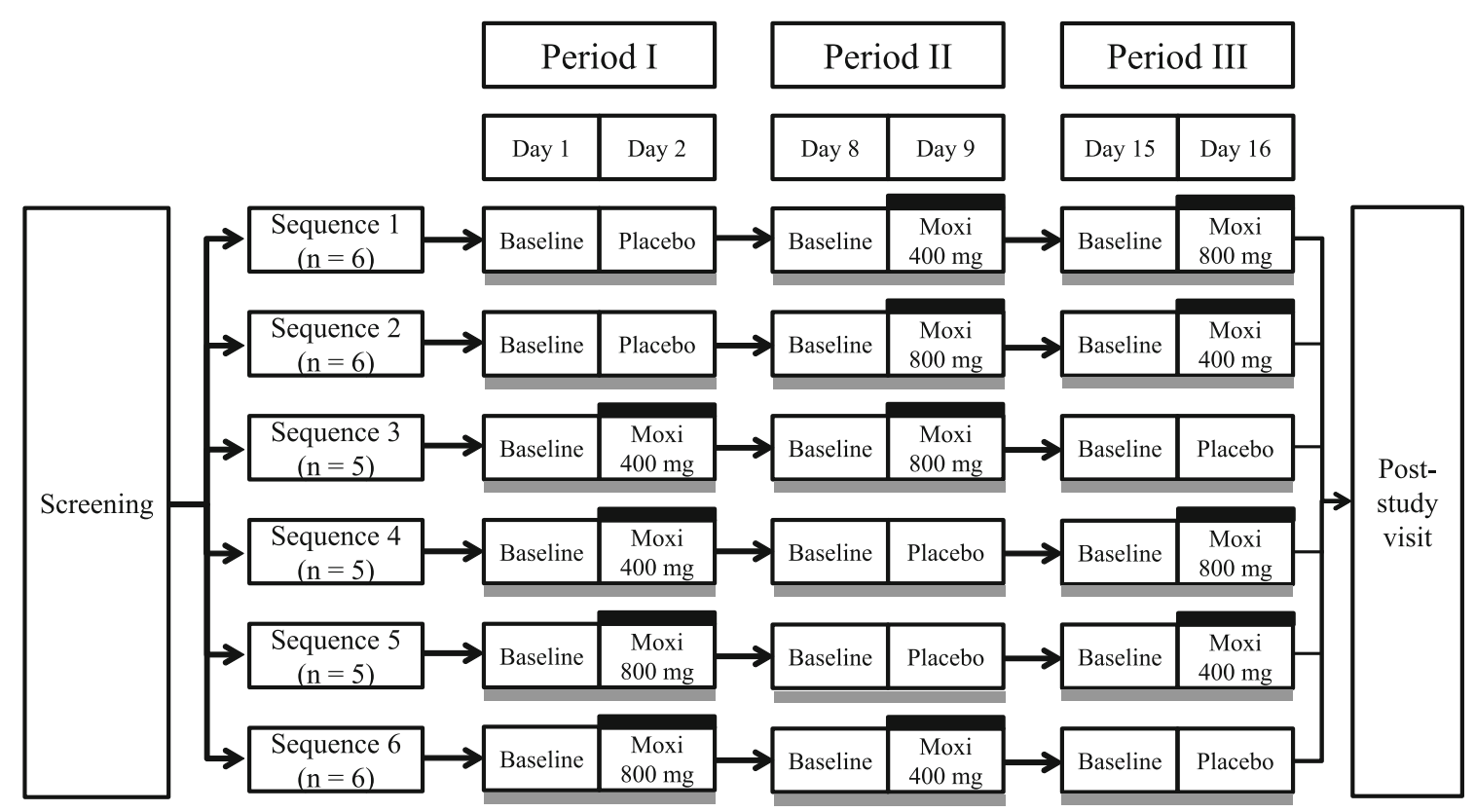

Fig. 1 Study design pharmacokinetic sampling (black shaded line); 12-lead electrocardiogram (grey shaded line)

Bayer Korea Ltd., Seoul, Korea), or moxifloxacin $800 \mathrm{mg}$. ECGs were then recorded at the corresponding time points in the same manner as on the baseline day. Blood sampling for the pharmacokinetic (PK) analyses was conducted at the same time points as the ECG recordings for subjects who took moxifloxacin. When the procedures were to be processed at the same time, the ECG was taken first, after which point, the vital signs were measured and the PK sampling was conducted to minimize the influence of the other procedures on the ECG results. The plasma was immediately separated by centrifugation at $2,093 \times g$ for $10 \mathrm{~min}$ at $4{ }^{\circ} \mathrm{C}$ and was stored at $-70{ }^{\circ} \mathrm{C}$ until further analysis. A washout period of 7 days was selected on the basis of the terminal half-life and the effects of moxifloxacin on the QT interval [4]. To minimize variability among the three study centers, each center used the same bottled water (Volvic, Group Danone S.A., Paris, France) for drug administration and the same meal plans. To minimize variability between the ECG recording periods, the exact placement of landmarks (e.g., clavicle, nipples, and sternal notch) and precordial electrodes were marked on a transparent plastic film for each subject during Period I, and this film was used throughout the study for each individual subject. The exact places of precordial electrodes did not change over the whole course of the study.

The study protocol was approved by the institutional review boards at Seoul St. Mary's Hospital, Seoul National University Hospital, and Seoul National University Bundang Hospital. Each center was limited to the investigation of 12 subjects. All of the procedures were performed in accordance with the recommendations of the Declaration of Helsinki regarding biomedical research involving human subjects and the Korean Good Clinical Practice guidelines. This study was registered in the public registry at ClinicalTrials.gov (NCT01756521).

\subsection{Pharmacodynamic Analyses}

QT intervals were measured automatically using the MUSE CV information system (GE Medical Systems, Milwaukee, WI, USA) and the representative median value from 12 leads was taken. For all other values, including heart rate, PR interval, RR interval, and QRS interval, automatically calculated values from the MAC $5000^{\circledR}$ or MAC5500 ${ }^{\circledR}$ were used. The baseline-corrected difference in QTc $(\Delta \mathrm{QTc})$ and the placebo-adjusted difference in $\Delta \mathrm{QTc}(\Delta \Delta \mathrm{QTc})$ were calculated using either Bazett's formula $\left(\mathrm{QTcB}=\mathrm{QT} / \mathrm{RR}^{1 / 2}\right)$, Fridericia's formula $(\mathrm{QTcF}=$ $\mathrm{QT} / \mathrm{RR}^{1 / 3}$ ), or an individual QT/RR linear regression model (QTcI). This was performed by first correcting the QT interval, then calculating $\Delta \mathrm{QTc}$ and $\Delta \Delta \mathrm{QTc}$ as follows: $\Delta \mathrm{QTc}=\mathrm{QT}$ (Day 2) $-\mathrm{QT}$ (baseline) and $\Delta \Delta \mathrm{QTc}=$ $\Delta \mathrm{QTc}$ (treatment) $-\Delta \mathrm{QTc}$ (placebo). Individual corrections were performed using an approach described by Desai et al. [7]: First, the QT interval vs. RR interval data obtained from each subject were plotted and fitted to a linear mixed model using the equation $\log Q T_{i j}=B_{i}+$ $\alpha_{i} \log R R_{i j}+e_{i j}$, where $e^{B_{i}}$ is the subject-specific QT in seconds when the RR interval was $1 \mathrm{~s}, \alpha_{i}$ is the slope of the log-transformed RR vs. QT relationship, and $e_{i j}$ is an error term. The subscripts $i$ and $j$ refer to the individual $(i)$ and the measurement time $(j)$. This linear model was 
manipulated to yield a correction of the equation: $\mathrm{QTcI}=Q T /(R R)^{\alpha_{i}}$. This correction from the placebo phase was applied to the data obtained during each subject's treatment phases. A repeated-measures analysis of variance taking the baseline QTc (1d) as the covariate, and period, sequence, study site, dosing amount, and time as fixed effects, was used for the statistical analyses.

A linear model was used to evaluate the relationship between moxifloxacin concentration and $\Delta \Delta \mathrm{QTc}$. The slopes and intercepts were estimated using $\Delta \Delta \mathrm{QTc}$ calculated by Bazett's formula, Fridericia's formula, and the individual linear regression method.

In the present study, the time-matched baseline measurement was used in all QT interval calculations. However, to assess the differences between baseline measurement methods, an arbitrary pre-dose baseline at $0 \mathrm{~h}$ was chosen and $\Delta \mathrm{QTc}$ and $\Delta \Delta \mathrm{QTc}$ were calculated from it.

\subsection{Moxifloxacin Plasma Concentration Determinations}

The plasma concentrations of moxifloxacin were determined using API 3200 LC/MS/MS System (Applied Biosystems, Foster City, CA, USA). A volume of $200 \mu \mathrm{L}$ of plasma was deproteinized with $200 \mu \mathrm{L}$ of $10 \%$ trichloroacetic acid containing the internal standard (moxifloxacin-d4, $5 \mu \mathrm{g}$ / $\mathrm{mL}$ ). Fifty microliters of the supernatant was diluted with $450 \mu \mathrm{L}$ of distilled water and $5 \mu \mathrm{L}$ of the dilution was injected onto a Hypersil Gold C18 column $(50 \times 3.0 \mathrm{~mm}$, $5 \mu \mathrm{m}$ ) at a flow rate of $0.4 \mathrm{~mL} / \mathrm{min}$ under isocratic conditions with $35 \%$ methanol containing $0.1 \%$ formic acid. Analytes were detected using multiple-reaction monitoring in the electrospray positive-ionization mode of MS. The mass transitions were $m / z, 402.1 \rightarrow 384.0$ for moxifloxacin and $\mathrm{m} /$ $z 406.2 \rightarrow 388.2$ for the internal standard.

The lower limit of quantification was $100 \mathrm{ng} / \mathrm{mL}$. The intra- and inter-day precisions (relative standard deviation) were below $3.94 \%$ and the accuracy range was 97.73-106.6\%.

\subsection{Pharmacokinetic Analyses}

The following PK parameters were assessed using a noncompartmental method with Phoenix WinNonlin ${ }^{\circledR}$ (Pharsight, Mountain View, CA, USA): maximum observed drug concentration $\left(C_{\max }\right)$, time to reach $C_{\max }$ following drug administration $\left(T_{\max }\right)$, area under the plasma concentration-time curve (AUC) from $0 \mathrm{~h}$ to the last measurable concentration $\left(\mathrm{AUC}_{\text {last }}\right)$, AUC from $0 \mathrm{~h}$ extrapolated to infinite time $\left(\mathrm{AUC}_{\mathrm{inf}}\right)$, terminal elimination half-life $\left(t_{1 /}\right.$ $2)$, apparent clearance (CL/F), and apparent volume of distribution $(\mathrm{Vd} / \mathrm{F}) . C_{\max }$ and $T_{\max }$ were determined by direct inspection of individual $\mathrm{PK}$ data, whereas $\mathrm{AUC}_{\text {last }}$ and $\mathrm{AUC}_{\text {inf }}$ were calculated using the linear up/log-down method. These parameters were compared between treatments (moxifloxacin 400 and $800 \mathrm{mg}$ ).

\subsection{Safety Assessments}

The safety of subjects was assessed via vital sign measurements, physical examinations, adverse events, clinical laboratory tests, and 12-lead ECG. Subjects were asked open-ended questions about their well-being, and adverse events were recorded and assessed based on their number of occurrences, the number of subjects who experienced adverse events, and their severity, seriousness, and causal relationship to moxifloxacin.

\section{Results}

\subsection{Subject Demographics}

A total of 38 subjects were enrolled in the study. Five subjects withdrew consent prior to the completion of the study and 33 subjects completed the study. The means \pm standard deviation of subject demographic parameters were as follows: age $26.4 \pm 4.8$ years, height $174.5 \pm 5.0 \mathrm{~cm}$, weight $68.3 \pm 6.3 \mathrm{~kg}$, and baseline QTcF $398.3 \pm 16.1 \mathrm{~ms}$. There were no statistically significant differences in demographic characteristics (age, height, weight, and baseline QTcF interval) among the sequence groups and study centers (data not shown).

\subsection{Pharmacodynamic Analyses}

There were definite increases in $\Delta \Delta \mathrm{QTc}$ after moxifloxacin dosing (Fig. 2). $\Delta \Delta$ QTcI at each measurement time is shown, with means and two-sided $90 \%$ confidence intervals (CIs) that did not include zero at any of the measurement times (Table 1). The majority of the largest timematched $\Delta \Delta \mathrm{QTc}$ occurred approximately $4 \mathrm{~h}$ after dosing (Table 2). Depending on the correction method used to calculate QTc, moxifloxacin $400 \mathrm{mg}$ prolonged the QT interval from $12 \mathrm{~ms}(\mathrm{QTcI})$ to $16 \mathrm{~ms}(\mathrm{QTcB})$ and moxifloxacin $800 \mathrm{mg}$ prolonged QTc from $21 \mathrm{~ms}$ (QTcI) to $29 \mathrm{~ms}$ (QTcB).

An increase in plasma moxifloxacin concentration was weakly associated with QTc prolongation (Fig. 3). The slopes of the regression lines using each correction method differed slightly $(\Delta \Delta \mathrm{QTcB}: 0.0067, \Delta \Delta \mathrm{QTcF}: 0.00535$, and $\Delta \Delta$ QTcI: 0.0047$)$, while the correlation coefficients were similar for each correction method $(\Delta \Delta \mathrm{QTcB}$ : 0.4344, $\Delta \Delta$ QTcF: 0.4346 , and $\Delta \Delta$ QTcI: 0.4220 ). There was a statistically significant difference between the 
A
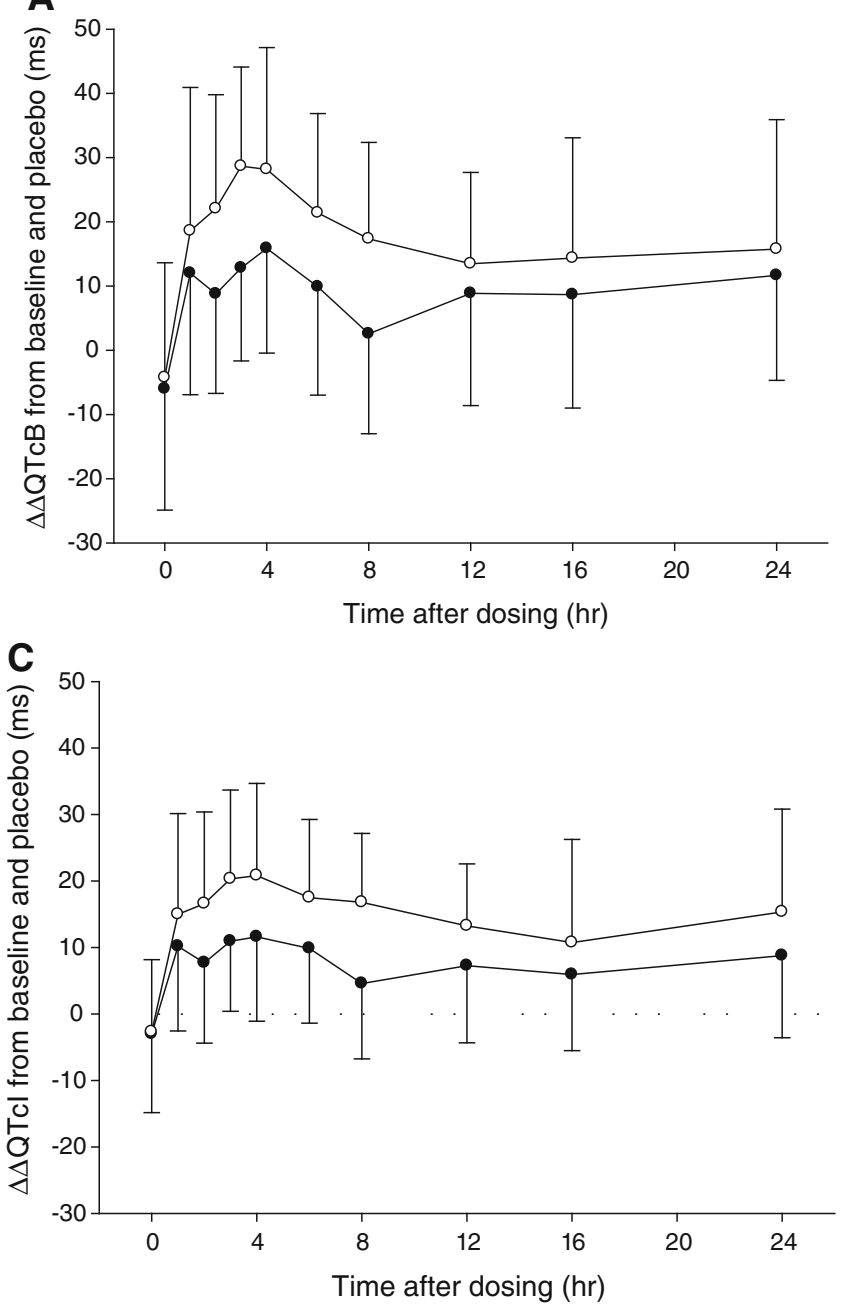

B

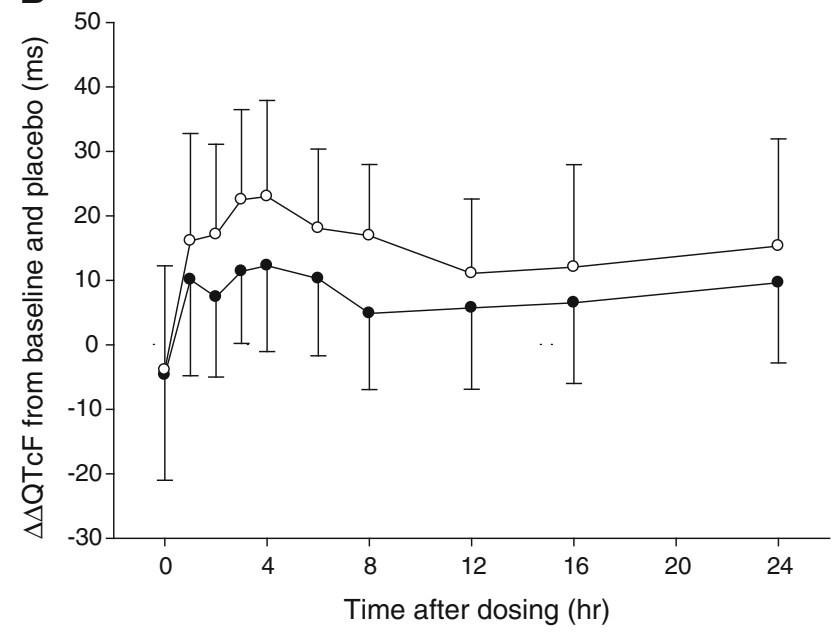

Fig. 2 Baseline- and placebo-corrected QT ( $\Delta \Delta \mathrm{QTC})$-time profiles using: a Bazett's formula, b Fridericia's formula, and $\mathbf{c}$ the individually corrected method. The data are presented as the arithmetic means \pm standard deviation (solid circle $400 \mathrm{mg}$, open circle $800 \mathrm{mg}$ )

Table 1 Baseline- and placebo-adjusted QTcI (QT interval corrected by individual QT-RR regression) mean differences and $90 \%$ confidence intervals by time point $(\mathrm{ms})$

\begin{tabular}{|c|c|c|c|c|c|c|}
\hline \multirow{3}{*}{$\begin{array}{l}\text { Time } \\
\text { (h) }\end{array}$} & \multicolumn{6}{|c|}{ Treatment } \\
\hline & \multicolumn{3}{|c|}{ Moxifloxacin $400 \mathrm{mg}$} & \multicolumn{3}{|c|}{ Moxifloxacin $800 \mathrm{mg}$} \\
\hline & Mean & $\begin{array}{l}90 \% \\
\text { lower }\end{array}$ & $\begin{array}{l}90 \% \\
\text { upper }\end{array}$ & Mean & $\begin{array}{l}90 \% \\
\text { lower }\end{array}$ & $\begin{array}{l}90 \% \\
\text { upper }\end{array}$ \\
\hline 1 & 10.23 & 6.66 & 13.79 & 15.13 & 11.57 & 18.70 \\
\hline 2 & 7.74 & 4.18 & 11.30 & 16.73 & 13.17 & 20.30 \\
\hline 3 & 10.99 & 7.43 & 14.55 & 20.46 & 16.90 & 24.02 \\
\hline 4 & 11.66 & 8.10 & 15.22 & 20.96 & 17.40 & 24.53 \\
\hline 6 & 9.90 & 6.34 & 13.46 & 17.64 & 14.08 & 21.20 \\
\hline 8 & 4.63 & 1.07 & 8.19 & 16.93 & 13.37 & 20.49 \\
\hline 12 & 7.32 & 3.76 & 10.88 & 13.40 & 9.83 & 16.96 \\
\hline 16 & 5.98 & 2.41 & 9.54 & 10.88 & 7.32 & 14.44 \\
\hline 24 & 8.82 & 5.25 & 12.38 & 15.49 & 11.93 & 19.05 \\
\hline
\end{tabular}

Table 2 Largest time-matched $\Delta \Delta \mathrm{QTc}$ (baseline- and placeboadjusted corrected QT) by treatment. Least-squares mean difference adjusted by placebo [90\% confidence intervals (CI)]

\begin{tabular}{lllll}
\hline & \multicolumn{2}{l}{ Treatment } \\
\cline { 2 - 3 } & \multicolumn{2}{l}{ Moxifloxacin $400 \mathrm{mg}$} & & \multicolumn{2}{l}{ Moxifloxacin $800 \mathrm{mg}$} \\
\cline { 2 - 3 } $\begin{array}{l}\text { Time } \\
\text { (h) }\end{array}$ & Mean $[90 \% \mathrm{CI}]$ & & $\begin{array}{l}\text { Time } \\
(\mathrm{h})\end{array}$ & Mean $[90 \% \mathrm{CI}]$ \\
\hline QTcB & 4 & $15.95[10.81$, & 3 & $28.83[23.69$, \\
& & $21.09]$ & & $33.97]$ \\
QTcF & 4 & $12.31[8.38$, & 4 & $23.14[19.21$, \\
& & $16.24]$ & & $27.07]$ \\
QTcI & 4 & $11.66[8.10$, & 4 & $20.96[17.40$, \\
& & $15.22]$ & & $24.53]$ \\
\hline
\end{tabular}

$\overline{Q T C B}$ corrected QT using Bazett's formula, $Q T c F$ corrected QT using Fridericia's formula, $Q T c I$ corrected QT using individual QT/RR regression model 
time-matched and pre-dose baseline measurement methods (Fig. $4, P<0.001$ ), but the time courses of the $\Delta \Delta \mathrm{QTc}$ profiles were similar between the two baseline correction methods $(P=0.853)$. QTcI regression showed rate-correction coefficient $(\alpha)$ values of $0.305 \pm 0.044$ (mean and standard deviation), with a minimum value of 0.207 and a maximum value of 0.413 (data not shown), which is comparable to the $\alpha$ value of QTcF (0.333).

Differences among study centers, sequence groups, periods, and treatment-time interaction did not influence the variation in QTc prolongation (data not shown). QTc prolongation was affected by the different treatments, (i.e., moxifloxacin 400 or $800 \mathrm{mg}$ ) and by time (both $P<0.0001$ ).

\subsection{Pharmacokinetic Analyses}

Dose-dependent PK profiles were observed in the moxifloxacin concentration-time profiles (Fig. 5). The
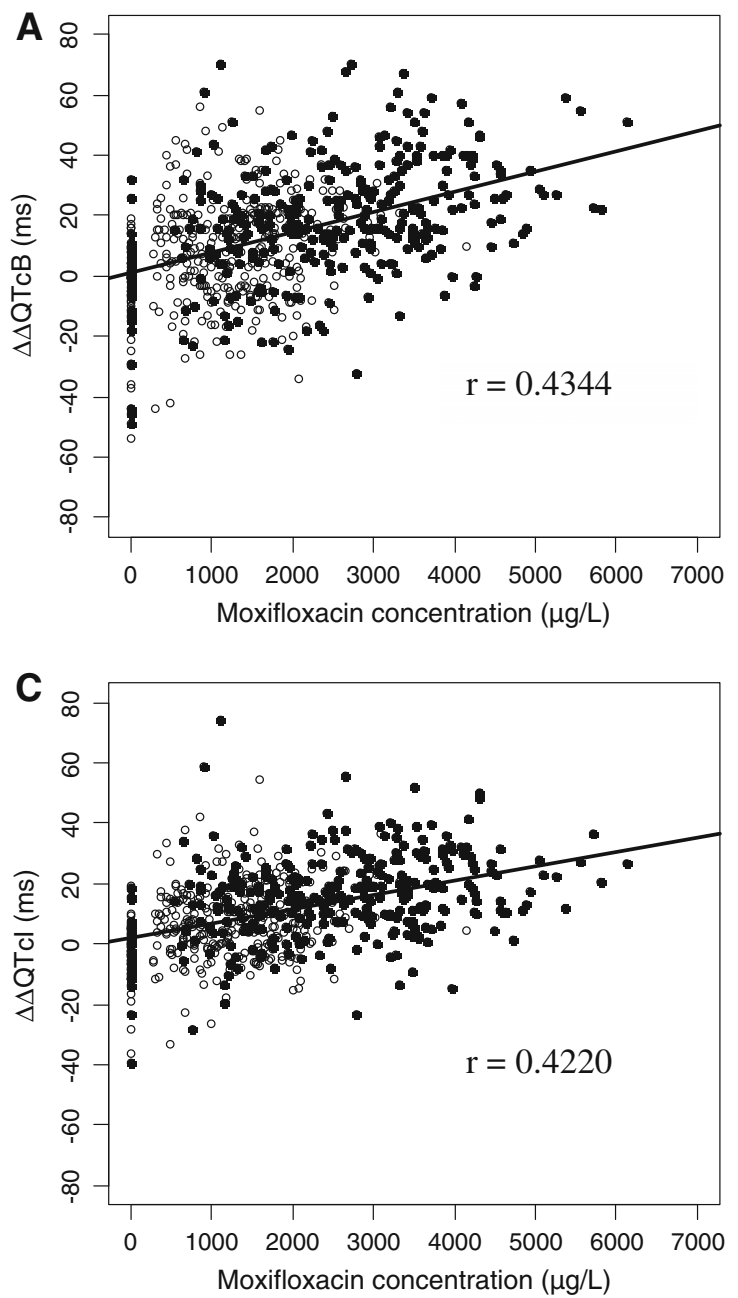

Fig. 3 Plasma concentrations of moxifloxacin vs. corrected QT $(\Delta \Delta \mathrm{QTc})$ scatter plot and regression lines using: a Bazett's formula, b Fridericia's formula, and $\mathbf{c}$ the individual correction method. The equations for each regression line were as follows: median value for $T_{\max }$ was slightly greater in the moxifloxacin 800-mg group than in the moxifloxacin 400-mg group. Certain parameters, such as $t_{1 / 2}, \mathrm{CL} / \mathrm{F}$, and $\mathrm{Vd} / \mathrm{F}$ did not significantly differ between the treatment groups, while other parameters, such as $C_{\max }$ and $\mathrm{AUC}_{\text {last }}$, tended to increase two-fold as the dose doubled (data not shown).

\subsection{Safety Assessments}

A total of 14 subjects reported 11 adverse events, which included chest discomfort, chill, diarrhea, dizziness, dry mouth, epistaxis, fever, nausea, paresthesia, pruritis, and rhinorrhea. Among these, chest discomfort, diarrhea, and nausea were assessed to be either possibly or probably related to moxifloxacin. No serious adverse events were reported and all of the reported adverse events disappeared spontaneously.

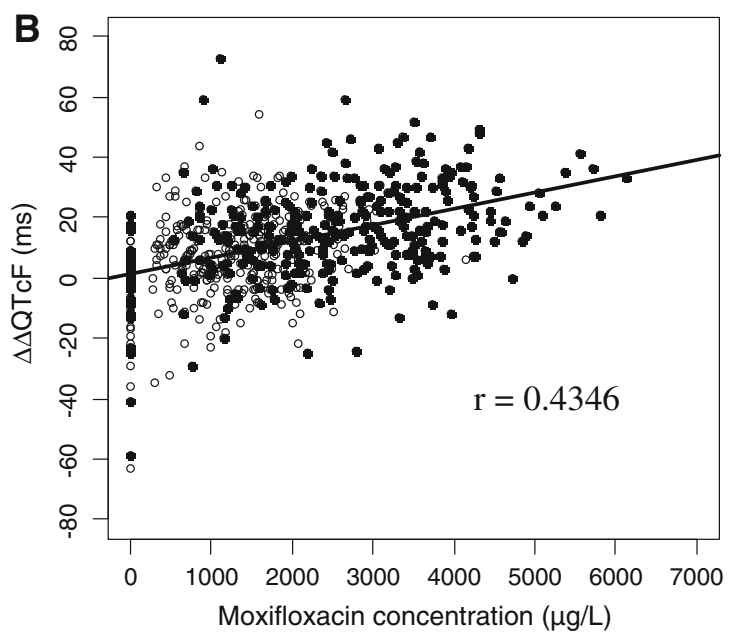

a $\Delta \Delta \mathrm{QTcB}=0.901+0.00670 \times$ moxifloxacin concentration, b $\Delta \Delta \mathrm{QTcF}=1.39+0.00535 \times$ moxifloxacin concentration, and c $\Delta \Delta \mathrm{QTcI}=2.36+0.00470 \times$ moxifloxacin concentration (open circle $400 \mathrm{mg}$, solid circle $800 \mathrm{mg}$ ) 

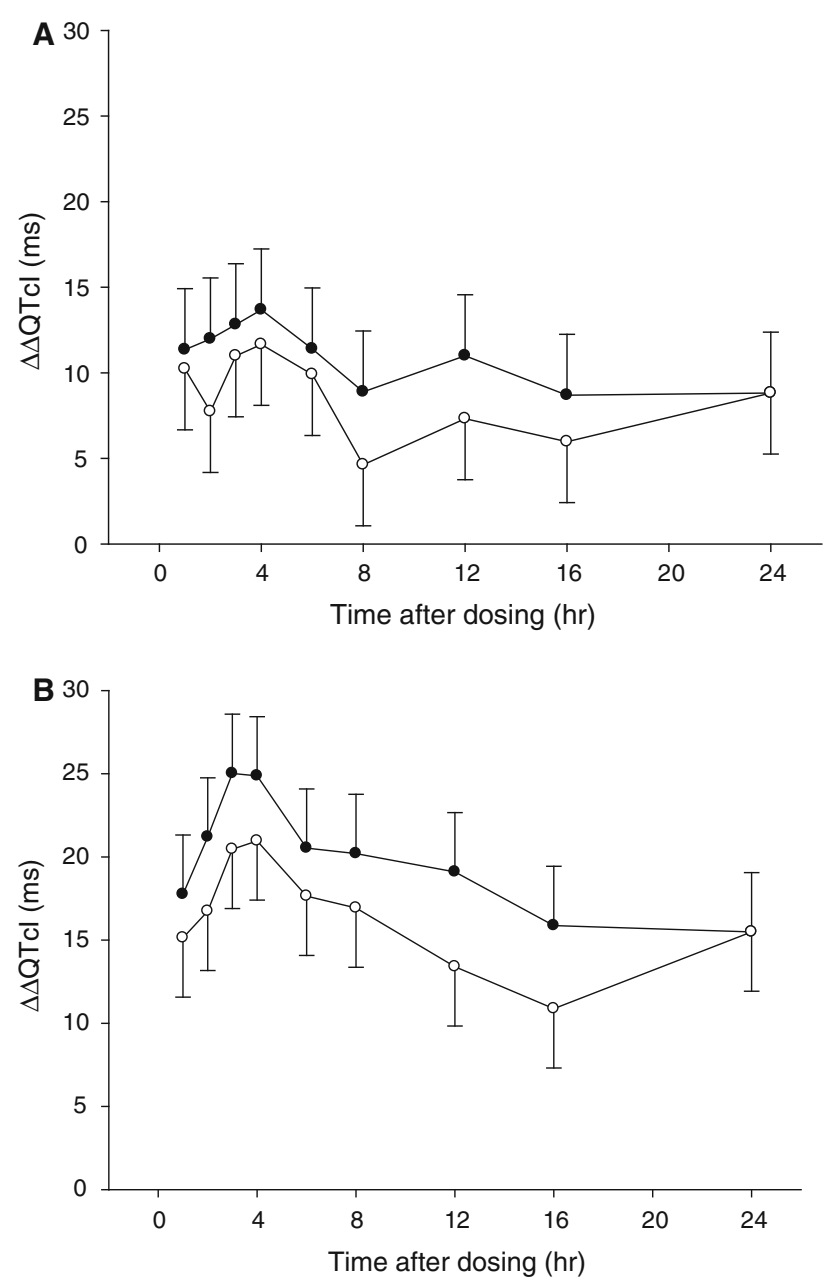

Fig. 4 Comparison of pre-dose baseline-corrected (solid circle) and time-matched (open circle) $\Delta \Delta \mathrm{QTCI}$ (mean differences with $90 \%$ confidence intervals) in a the moxifloxacin 400-mg group and $\mathbf{b}$ the moxifloxacin 800-mg group

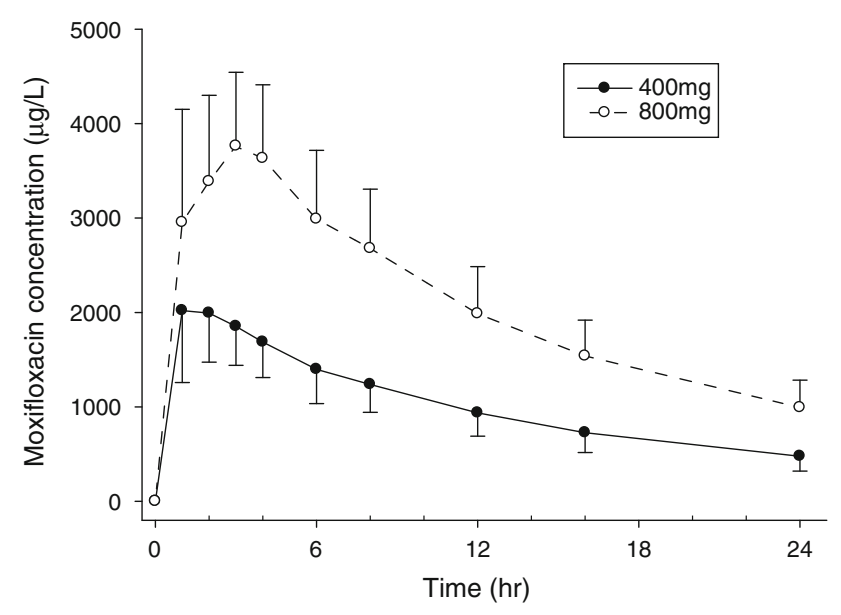

Fig. 5 Plasma concentration-time profiles after a single oral administration of moxifloxacin

\section{Discussion}

Our study found a definite prolongation of the QTc interval after moxifloxacin dosing [11.66 $\mathrm{ms}$ in the moxifloxacin 400-mg group and $20.96 \mathrm{~ms}$ in the moxifloxacin $800-\mathrm{mg}$ group (QTcI values)]. The mean differences and $90 \%$ CIs of $\Delta \Delta \mathrm{QTcI}$ did not include zero at any of the measurement time points. A positive relationship between QT interval prolongation and moxifloxacin concentration $(r=0.422$ in $\Delta \Delta \mathrm{QTcI}$ ) was also observed. The $T_{\max }$ of moxifloxacin 400 and $800 \mathrm{mg}$ occurred 1 and $3 \mathrm{~h}$ after dosing, respectively, whereas the largest time-matched $\Delta \Delta \mathrm{QTc}$ was measured approximately $4 \mathrm{~h}$ after dosing.

Moxifloxacin $400 \mathrm{mg}$ is known to cause a mean increase in the QTc interval of between 10 and $14 \mathrm{~ms} 2-4 \mathrm{~h}$ after a single oral dose $[4,8]$, which was consistent with the results of this study. In addition, a supratherapeutic dose of moxifloxacin $(800 \mathrm{mg})$ resulted in a nearly 2 -fold increase in the QTc interval from baseline compared with the 400-mg dose, which was greater than the previous report by Demolis et al. [4]. Although Demolis et al. only used QTcB and QTcF values in their study, they found no relationship between the dose of moxifloxacin and QT interval lengthening, but found a positive relationship between QT interval prolongation and moxifloxacin concentration $[r=0.35$, moxifloxacin concentration $(\mu \mathrm{g} / \mathrm{L})$ vs. QT interval (ms); $r=0.72$, moxifloxacin concentration $(\mu \mathrm{g} / \mathrm{L})$ vs. $\Delta \mathrm{QT}$ interval $(\%)]$. The study that was conducted by Demolis et al. differs from our study in that they performed submaximal exercise testing to allow for variation in RR intervals, which may explain the differences between the correlation coefficients (moxifloxacin concentration vs. QT or QTc interval) in the two studies. Their findings with supratherapeutic doses of moxifloxacin differed from those of our study, in which an increase in the moxifloxacin dose almost doubled $\Delta \Delta \mathrm{QT}$ c. Because there were no noticeable differences in PK parameters between the two studies, there is a possibility that Korean subjects may show different susceptibility to supratherapeutic doses of moxifloxacin than Caucasian subjects. Nonetheless, our findings suggest that moxifloxacin induces a detectable effect of greater than $5 \mathrm{~ms}$ on QTc prolongation, which confirms the adequacy of the use of moxifloxacin as a positive control in Korean TQT studies, explained by Answer 1 in the ICH E14 Questions-and-Answers document [9].

Data reported by Florian et al. [8] showed the sufficiency of linear concentration- $\Delta \Delta \mathrm{QTCF}$ model in describing the effect of moxifloxacin on QT interval. Pooled data from $20 \mathrm{TQT}$ studies were analyzed, and a mean slope of $3.1 \mathrm{~ms}$ per $\mu \mathrm{g} / \mathrm{mL}$ was estimated. This estimated slope is 
smaller when compared with the present study's slope ( $0.00535 \mathrm{~ms}$ per $\mu \mathrm{g} / \mathrm{L}$ for $\Delta \Delta \mathrm{QTcF})$. Although Caucasians were more than $80 \%$ of the dataset in Florian et al., it is unlikely that this difference is because of ethnicity. There seems to be a wide inter-individual variability in moxifloxacin-induced QT response, as the range of the slope varied greatly from 1.6 to $4.8 \mathrm{~ms}$ per $\mu \mathrm{g} / \mathrm{mL}$ even when the percentages of ethnic backgrounds were similar between studies. Therefore, the difference in mean slopes of concentration- $\Delta \Delta \mathrm{QT}$ c models is likely because of individual variability.

A study that recruited healthy Japanese subjects [10], which reported the largest QTcF change from baseline as $11.6 \mathrm{~ms}(90 \% \mathrm{CI}$ 9.1-14.1) in a non-fasting state and $14.4 \mathrm{~ms}$ (90 \% CI 11.9-16.8) in a fasting state, found no statistically significant differences between Caucasian and Japanese subjects in QTc interval prolongation. The value obtained in the fasting state was similar to the largest $\Delta \Delta \mathrm{QTcF}$ found in our study, but because direct comparison is not possible, this does not imply ethnic differences between Japanese and Korean subjects. It is worth noting, however, that there was a study (ClinicalTrials.gov identifier NCT01876316) that compared moxifloxacin-induced QT prolongation in Japanese and Korean subjects, and this study has concluded there was no significant difference between the two ethnicities (unpublished data).

One study that used a quinidine intravenous infusion to evaluate differences in QT interval prolongation between Caucasian and Korean subjects reported that Korean subjects were less sensitive to quinidine-induced QT prolongation than Caucasian subjects [11]. Although this study contributed valuable Korean QT prolongation study data, a difference exists: this study did not use moxifloxacin, a drug that is commonly used as a positive control in TQT studies.

Previously identified differences based on QT interval correction methods were observed [6]: namely, the tendency of Bazett's formula to extend to extreme values. This tendency was more evident in the moxifloxacin 800-mg group, where the largest time-matched $\Delta \Delta \mathrm{QTcB}$ was calculated to be $28.83 \mathrm{~ms}$ (90\% CI 23.69-33.97). Therefore, a correction method using either Fridericia's formula or individual correction may be a better choice for TQT studies in Korean subjects, where individual correction would most likely be the best choice as noted previously [1]. We also investigated different baseline measurement methods and found a statistically significant difference between two baseline measurement methods; namely, a trend was observed in which the $\Delta \Delta \mathrm{QTc}$ from the time-matched baseline was measured to be lower than that from the pre-dose baseline. This trend did not change over time. This finding may be because the time-matched baseline measurement corrects for diurnal variation. One limitation to our study is the fact we took only one pre-dose recording, while the usual pre-dose baseline measurement is conducted by taking the median QTc value from three pre-dose ECG recordings [9]. Therefore, an exact one-onone comparison of the time-matched and pre-dose baseline methods was not appropriate. ICH guideline E14 recommends that parallel studies use the time-matched baseline method and that crossover studies use the pre-dose baseline method [9]. In contrast to the recommendations, our study was a crossover study that used the time-matched baseline method; however, despite the identified limitations of our study, we think that the time-matched baseline measurement can also be used in crossover studies because of its merits in diurnal variation correction.

A study by Yan et al. [12] suggested that parallel studies using time-matched baseline correction could show higher variation in $\triangle \mathrm{QTcF}$ and result in smaller correlation, probably because of a time lag between baseline measurement and dosing. Yan et al. have also found slightly lower values for $\Delta \Delta \mathrm{QTCF}$ in crossover designs that used pre-dose baseline correction. Because our study is unique in that we have set up a crossover study with time-matched baseline method, it is quite difficult to compare whether one baseline correction method is preferable in place of another. At present, there could be discrepancies between studies analyzing different correction methods.

We speculated that by confirming the QT interval prolongation effects of moxifloxacin we could obtain comparable pilot data that could be used in QT interval prolongation studies in drug development targeting the Korean population. By doing so, an effective safety monitoring plan can be established and guidelines for accurate ECG data collection and analysis may be presented. Bazett's formula is not recommended for calculating the range of QT interval prolongation corrected by individual RR, but Fridericia's formula and the individual correction method may be used interchangeably. The current study aimed to provide comparable pilot QT interval prolongation data in Korean subjects that could be used in scientific and regulatory fields and was not necessarily focused on detecting inter-ethnic differences. However, because other studies have suggested that possible differences exist between ethnic groups, further studies are needed to evaluate and incorporate possible interethnic differences. In addition, because this study only included male subjects, gender differences were not evaluated.

\section{Conclusion}

In summary, moxifloxacin $400 \mathrm{mg}$ causes moderate QT interval prolongation and is an adequate positive control in Korean TQT studies. Our results indicate that caution 
should be exercised when a supratherapeutic dose of moxifloxacin is used in Korean subjects. Furthermore, the findings of the present study may be employed in drug development studies targeting the Korean population and may also be applied to further research attempting to evaluate the cardiac safety of a drug in Korean subjects.

Acknowledgments This study was supported by a grant from the Korea Healthcare Technology R\&D Project, Ministry of Health \& Welfare, Republic of Korea (HI07C00010000, Korea National Enterprise for Clinical Trials). The authors would like to thank HyoBum Seo for the analyses of moxifloxacin concentration and Jewon Lee for the manual measurements of ECG recordings. The authors do not have any conflicts of interest to declare.

Open Access This article is distributed under the terms of the Creative Commons Attribution Noncommercial License which permits any noncommercial use, distribution, and reproduction in any medium, provided the original author(s) and the source are credited.

\section{References}

1. International Conference on Harmonisation. The clinical evaluation of QT/QTc interval prolongation and proarrhythmic potential for non-antiarrhythmic drugs (ICH E14). ICH, Geneva, 12 May 2005. Available at: http://www.ich.org/fileadmin/Public_ Web_Site/ICH_Products/Guidelines/Efficacy/E14/E14_Guideline. pdf Accessed 03 Jan 2014.

2. Haverkamp W, Breithardt G, Camm AJ, et al. The potential for QT prolongation and pro-arrhythmia by non-anti-arrhythmic drugs: clinical and regulatory implications. Report on a Policy Conference of the European Society of Cardiology. Cardiovasc Res. 2000;47(2):219-33.

3. Malik M, Garnett CE, Zhang J. Thorough QT studies: questions and quandaries. Drug Saf Int J Med Toxicol Drug Exp. 2010;33(1):1-14.
4. Demolis JL, Kubitza D, Tenneze L, Funck-Brentano C. Effect of a single oral dose of moxifloxacin (400 $\mathrm{mg}$ and $800 \mathrm{mg}$ ) on ventricular repolarization in healthy subjects. Clin Pharmacol Ther. 2000;68(6):658-66.

5. Shah RR. Drug-induced QT interval prolongation: does ethnicity of the thorough QT study population matter? Br J Clin Pharmacol. 2013;75(2):347-58.

6. Malik M, Farbom P, Batchvarov V, Hnatkova K, Camm AJ. Relation between QT and RR intervals is highly individual among healthy subjects: implications for heart rate correction of the QT interval. Heart. 2002;87(3):220-8.

7. Desai M, Li L, Desta Z, Malik M, Flockhart D. Variability of heart rate correction methods for the QT interval. Br J Clin Pharmacol. 2003;55(6):511-7.

8. Florian JA, Tornoe CW, Brundage R, Parekh A, Garnett CE. Population pharmacokinetic and concentration-QTc models for moxifloxacin: pooled analysis of 20 thorough QT studies. J Clin Pharmacol. 2011;51(8):1152-62.

9. International Conference on Harmonisation. E14 Implementation Working Group. ICH E14 Guideline: the clinical evaluation of QT/QTc interval prolongation and proarrhythmic potential for non-antiarrhythmic drugs: questions and answers (R1). ICH, Geneva, 5 April 2012. Available at: http://www.ich.org/fileadmin/ Public_Web_Site/ICH_Products/Guidelines/Efficacy/E14/E14_Q_ As_R1_step4.pdf. Accessed 03 Jan 2014.

10. Taubel J, Ferber G, Lorch U, Batchvarov V, Savelieva I, Camm AJ. Thorough QT study of the effect of oral moxifloxacin on QTc interval in the fed and fasted state in healthy Japanese and Caucasian subjects. Br J Clin Pharmacol. 2014;77(1):170-9.

11. Shin JG, Kang WK, Shon JH, et al. Possible interethnic differences in quinidine-induced QT prolongation between healthy Caucasian and Korean subjects. Br J Clin Pharmacol. 2007; 63(2):206-15.

12. Yan LK, Zhang J, Ng MJ, Dang Q. Statistical characteristics of moxifloxacin-induced QTc effect. J Biopharm Stat. 2010;20(3): 497-507. 